\title{
O uso da música para a regulação do estado de ânimo no período pós-COVID-19
}

\author{
João Fortunato Soares-Quadros Junior \\ Universidade Federal do Maranhão (Brasil) \\ joaofjr@gmail.com \\ https://orcid.org/0000-0003-3763-929X \\ Carmen María Román-Torres \\ Universidad de Granada (Espanha) \\ carmen_cnl@hotmail.com \\ https://orcid.org/0000-0002-9381-4745 \\ Alberto Joaquim Gouveia Diniz Neto \\ Universidade Federal do Maranhão (Brasil) \\ albertodiniz1308@gmail.com \\ https://orcid.org/0000-0002-0728-6035 \\ Ivo Freitas Santana \\ Universidade Federal do Maranhão (Brasil) \\ ivofsantana@gmail.com \\ https://orcid.org/0000-0002-8020-5510
}

Resumo: A música tem sido apontada na literatura científica como um meio eficaz para a regulação do estado de ânimo. Partindo dessa premissa, buscamos nessa revisão apresentar com clareza a concepção sobre estado de ânimo, abordando os seus tipos e a sua relação com a música. Para tanto, foi realizada uma ampla pesquisa bibliográfica junto a publicações nacionais e internacionais de maior impacto. Além disso, buscou-se também contextualizar essa discussão considerando os impactos psicológicos negativos causados pela pandemia da COVID-19. Assim, observamos que existem certos tipos de música que tem demonstrado uma capacidade interessante para induzir estados de ânimo positivos, tais como o dance, a música clássica e o rap. Ademais, a literatura sugere que a participação em atividades de escuta ou performance musicais, tanto individual ou coletivamente, podem favorecer a redução de emoções negativas (como ansiedade e depressão) e, consequentemente, melhorar o estado de ânimo. Portanto, essas evidências indicam que a música pode ser concebida como um importante meio para combater os impactos psicológicos causados pela pandemia. Com isso, espera-se que esse estudo possa incentivar e contribuir para o desenvolvimento de novos estudos acerca da relação entre música e humor.

Palavras-chave: Música, Estado de ânimo, Emoção, COVID-19, Sars-CoV-2.

\section{Music as a mood regulator in the COVID-19 pandemic}

\begin{abstract}
Music has been identified as a relevant tool for regulating mood. Based on this, we carried out a literature review that aims to show the concept and types of mood, as well as their relationship with music. We conducted a wide bibliographic research that considered national and international publications with the greatest impact. Furthermore, we sought to create a contextualized discussion with the current health situation in the world caused by the COVID-19 pandemic. Thus, we noted that some types of music have an interesting capacity to induce positive mood, like dance, classical, and rap. In addition, the literature suggests that participating in musical listening and/or performance activities, both individual and collective, can provoke a reduction in negative emotions (such as anxiety and depression) and improve mood. Therefore, it possible to believe that music can be an important way to combat the negative psychological impact caused by the pandemic. Thereby, we hope that this study might encourage and contribute to the development of new research relating music and mood.
\end{abstract}

Keywords: Music, Mood, Emotion, COVID-19, Sars-CoV-2. 


\section{Introdução}

O presente artigo tem como objetivo principal realizar uma revisão da literatura científica acerca dos temas música e estado de ânimo, buscando conhecer de que maneira a música contribui para a regulação do humor. Para tanto, foi levada a cabo uma extensa pesquisa bibliográfica que congregou autores brasileiros e internacionais na intenção de definir, com a maior clareza possível, os conceitos basilares da proposta. Além disso, esse trabalho traz uma abordagem contextualizada com a situação atual no mundo, no tocante à pandemia causada pelo surgimento do vírus Sars-CoV-2 (popularmente conhecido como COVID-19 ou apenas Coronavírus). Dessa forma, espera-se que este estudo possa auxiliar pesquisadores no desenvolvimento de propostas que tenham, na música, um meio fundamental para amenização dos impactos psicológicos negativos ocasionados por essa doença.

\section{Definindo 'estado de ânimo'}

‘Estado de ânimo' é definido na literatura científica como um episódio difuso, de baixa intensidade, de longa duração e sem causa aparente (PERGHER et al., 2006). Segundo Dalgalarrondo (2018), isso é vivido corporalmente e tem relação considerável com as condições corporais e vegetativas do organismo, sendo um fenômeno essencial para o funcionamento da vida humana. Para esse autor, quando os estados de ânimo estão relacionados à estimulações externas, podem sofrer influência e alterar com facilidade os comportamentos do indivíduo. De acordo com Longhi, Behar e Bercht (2010), seus efeitos podem ser analisados a partir dos processos cognitivos de valência positiva (que são estímulos atrativos) ou negativa (que são estímulos repulsivos).

A compreensão acerca dos estados de ânimo está diretamente interligada com os estudos derivados dos estados emocionais, que fazem parte das dimensões que ajudam a entender o funcionamento humano (LONGHI; BEHAR; BERCHT, 2010). Os estudos que tratam dos estados emocionais são estruturados a partir de diferentes conceitos, tais como afetividade, emoção e sentimentos.

Conforme Dalgalarrondo (2018) sugere, a afetividade integra o rol das dimensões psíquicas, sendo responsável por dar "cor, brilho e calor" a todas as vivências humanas. De acordo com Fiorin (2008), esse construto engloba muitos conceitos importantes e 
distintos, tais como o humor ou estado de ânimo, as emoções e os sentimentos. Por vezes, esses conceitos são usados de forma similar no senso comum, mas, quando se trata de pesquisas ou intervenções, é fundamental demarcar suas diferenças. Estudos sobre esse tema têm se mostrado de grande relevância sobretudo com respeito à qualidade de interação na relação professor-aluno (ALMEIDA; 1999; LEITE; TASSONI, 2002). Apesar disso, ainda são escassas as produções científicas sobre afetividade dentro do ambiente escolar na literatura brasileira (NOGUEIRA; LEITE, 2015).

As emoções são definidas como reações afetivas momentâneas, de alta intensidade e curta duração, relacionadas a estímulos internos ou externos específicos, sendo eles conscientes, não conscientes ou inconscientes (GARRIDO, 2014; PERGHER et al., 2006). Para Oatley e Johnson-Laird (1987), emoções ajudam a dar sentido à existência humana, tendo em vista que a vida é condicionada e organizada por diferentes dimensões, tais como necessidades, motivações e preocupações. Segundo Frijda (1986), a cognição é a dimensão responsável pela qualificação e interpretação das alterações somatossensoriais, tais como alegria ou medo.

Nessa mesma perspectiva, Dalgalarrondo (2018) define os sentimentos como estados e configurações afetivas estáveis, de menor intensidade e menos reativos a estímulos passageiros (em comparação às emoções). Esse fenômeno está fortemente ligado aos processos mentais em comparação aos somáticos, tendo uma interferência maior com conteúdo intelectuais e da cultura. Há pontos importantes na compreensão dos sentimentos, pois eles são diferentes quando se pensa nas culturas e nos valores retratados. Por exemplo, as noções de amor, tristeza, luto e alegria são diferentes em vários países, bem como a forma de se comportar diante desses sentimentos, sendo esta mais orientada pelos costumes culturais, ao invés de um processo puramente interno ou um comportamento inato.

Por outro lado, existem evidências que sugerem uma relação direta entre humor e memória. Em seu estudo, Pergher et al. (2006) identificou dois tipos de fenômenos que ocorrem frequentemente no nosso dia-a-dia. O primeiro deles, denominado de memória congruente com o humor, é definido pelos autores como uma "tendência de codificar ou recordar materiais quando nos encontramos em um estado afetivo consistente com a valência afetiva desses conteúdos” (p. 62). Isso significa que um indivíduo em estado de 
ânimo positivo tende a recordar mais facilmente e em maior quantidade informações relacionadas a afetos positivos, em comparação com indivíduos em estado emocional negativo. O segundo fenômeno, chamado de memória dependente do humor, refere-se a "um aumento da probabilidade de o indivíduo lembrar materiais que foram aprendidos em um estado particular de humor" (p. 63). Por exemplo, caso um indivíduo escute uma música específica quando se encontre em um estado anímico triste, a tendência é de que ele recorde mais facilmente dessa música, sempre que estiver triste. Entretanto, os autores afirmam que ainda assim não é possível estabelecer de forma conclusiva uma relação causal entre estado de ânimo e memória, uma vez que os fenômenos observados durante o estudo produziram reações pontuais que estariam mais relacionadas a emoções, não ao estado de ânimo dos participantes.

A estreita relação entre emoção e estado de ânimo tem gerado grande confusão na literatura que se preza a desenvolver pesquisas sobre esses temas (ALI; PEYNIRCIOĞLU, 2006; ETZEL et al., 2006; ROY; PERETZ; RAINVILLE, 2008; SEIDEL; PRINZ, 2013). De acordo com Garrido (2014), é necessário que os pesquisadores adotem como parâmetro as seguintes quatro características: duração, grau de controle, intensidade e causa. Em comparação com os estados de ânimo, pode-se afirmar que as emoções são de curta duração, ocasionadas por um determinado estímulo (ou evento), de maior intensidade e menor grau de controle por parte dos indivíduos. Juslin e Sloboda (2010) as consideram mais flexíveis, ou seja, variam com maior rapidez no tempo e são de mais fácil modificação. Finalmente, Juslin e Västfjäll (2008) acreditam que uma diferença crucial entre esses termos está nos mecanismos pelos quais a música evoca certas respostas emocionais ou produz efeitos nos estados de ânimo. Segundo esses autores, os mecanismos implicados nas reações emocionais ante um estímulo musical são os reflexos do tronco encefálico, a indução rítmica, o contágio emocional, a imagem visual, a memória episódica, a expectativa musical e o juízo estético. Em contrapartida, os estados de ânimo induzidos pela música têm na reflexão, na catarse, no consolo/conexão, na ruminação e no prazer estético os seus principais mecanismos.

Apesar das diferenças conceituais estabelecidas entre os termos, eles compartilham componentes similares tais como reações fisiológicas, sentimentos subjetivos e expressões comportamentais (SAARIKALLIO, 2012). Por outro lado, é importante destacar que vários estudos indicam que a emoção pode ser convertida em estado de 
ânimo quando a origem que a causou é esquecida (ECHEVERRÍA, 2005). Além disso, o humor pode ser resultado de uma experiência emocional densa, sendo esta ativada com uma grande intensidade, de forma reiterada e em um curto espaço de tempo entre cada evocação (GALLARDO VERGARA, 2006).

\section{Tipos de estado de ânimo}

Todos os seres humanos, de forma individual, possuem um determinado estado de ânimo que não pode ser escolhido e nem controlado. Cada estado anímico impõe ao sujeito um padrão comportamental, condicionando suas ações e a forma pela qual estas são realizadas (ECHEVERRÍA, 2005). Cada indivíduo tende a possuir diferentes estados de ânimo, mas existem evidências que sugerem a possibilidade de que o humor possa ser induzido em função da afinidade interpessoal. Segundo Wróbel e Królewiak (2015), quando a relação entre indivíduos é agradável, é possível que haja uma indução para um estado de ânimo positivo. Entretanto, quando falta afinidade, essa indução tende a produzir estados de ânimo negativos.

Na literatura científica observa-se uma variada tipologia de estados de ânimo. Entretanto, Kouros e El-Sheikh (2015) sugerem uma organização em duas grandes categorias: estados de ânimo positivos e estados de ânimo negativos. O primeiro tipo está relacionado às sensações de felicidade, relaxamento, despreocupação e calma, enquanto que o segundo aparece relacionado a sentimentos de tristeza, tensão, irritação, preocupação, nervosismo ou a mudanças bruscas de humor. É importante ter em mente que o tipo de estado de ânimo de um indivíduo pode afetar consideravelmente a sua saúde mental e o seu bem-estar.

Dentro dos estados de ânimos positivos, o que mais se destaca na literatura é a alegria, algo que permite ao indivíduo sentir calma e desfrutar dos pequenos momentos do dia-a-dia (KOUROS; EL-SHEIKH, 2015). De acordo com Argyle e Martin (1991), as principais fontes de alegria para os seres humanos estão nas suas relações sociais (família e amigos), no êxito pessoal/profissional, na atividade sexual, no esporte, na leitura, na música, na dança, na comida e no consumo de álcool, tabaco e drogas ilícitas. Cada uma dessas fontes de alegria conduz, de alguma forma, a centros de prazer do indivíduo, sendo o sistema dopaminérgico um dos mais utilizados para essa finalidade (CUSTODIO; 
CANO-CAMPOS, 2017; ZATORRE; SALIMPOOR, 2013). De acordo com Boehm e Lyubomirsky (2008), a alegria permite que os indivíduos se adaptem melhor às mudanças, se sintam melhor consigo mesmos, experimentem emoções mais positivas e melhorem suas atividades acadêmicas e laborais. Assim, esse tipo de estado de ânimo possibilita aos sujeitos aumentarem sua sensação de felicidade, influenciando tanto sua saúde mental quanto sua qualidade de vida.

Por outro lado, um estado de ânimo positivo exerce enorme influência na forma de acesso aos pensamentos, sendo estes os primeiros que surgem na mente do indivíduo e que moldam as decisões que são tomadas no seu dia-a-dia. Assim, ao possuir um estado de ânimo positivo, o indivíduo tem acesso a uma grande quantidade de pensamentos positivos que podem facilitar avaliações cuidadosas, elaboradas e sistemáticas (ISEN, 2008). Além disso, a posse desse tipo de estado anímico melhora o processo cognitivo dos indivíduos (DJAMASBI; STRONG; DISHAW, 2010). Por conseguinte, Tamir e Robinson (2007) afirmam que os estados de ânimo positivos também facilitam atingir objetivos e resultados desejáveis, assim como melhorar as interações sociais.

Com relação aos estados de ânimo negativos, é importante salientar primeiramente que estes são os que mais aparecem mencionados nos estudos sobre o tema, podendo ser destacados a tristeza-depressão, a ansiedade e a ira. A tristeza-depressão, também definida como transtorno do estado de ânimo, pode ser ocasionada por baixos e contínuos níveis de estado anímico (SANZ, 2001). Ela é caracterizada como uma doença de caráter duradoura ou recorrente que aparece frequentemente associada a sintomas como tristeza, perda de interesse ou prazer, sentimentos de culpa ou baixa autoestima, sono ou apetite perturbados, sensação de cansaço, falta de concentração e diminuição da energia (BOTH et al., 2008; RUFINO et al., 2018). Essa doença pode gerar implicações significativas de ordem comportamental, laboral, social, afetivo e também de autoimagem (CAMPOS; DEL-PRETTE; DEL-PRETTE, 2014). Estima-se que atualmente mais de 320 milhões de pessoas no mundo tenham depressão (ORGANIZAÇÃO MUNDIAL DA SAÚDE, 2017).

A ira é concebida como um estado de ânimo frequentemente associado a sentimentos de irritação, tais como chateação, raiva e fúria (SANZ, 2001). Estes sentimentos variam de intensidade em função de cada pessoa e de cada situação, podendo ir desde uma pequena irritação a uma fúria intensa (DEL BARRIO; ALUJA; 
SPIELBERGER, 2009). De acordo com Lagos San Martín et al. (2015), a ira pode ser categorizada em dois tipos: ira-traço e ira-estado. A ira-traço é a predisposição natural que o indivíduo possui para experimentar, desenvolver e/ou expressar irritação sem a existência de um motivo específico. Por outro lado, a ira-estado se refere a experiências eventuais de sentimentos subjetivos como tensão, irritação ou fúria, que resultem de um momento particular e que pode vir acompanhado de aumentos da ativação fisiológica. Em resumo, é possível afirmar que o conceito de ira-traço evidencia o estado de ânimo negativo, enquanto que ira-estado faz referência a um tipo de estado emocional de curta duração e associado a diferentes variáveis psicológicas (OLIVA MENDOZA; HERNÁNDEZ POZO; CALLEJA BELLO, 2010).

A ansiedade, por sua vez, pode ser definida como uma reação emocional desagradável que gera tensão, desconforto e/ou pensamentos apreensivos, sendo uma resposta de defesa do nosso corpo frente a situações consideradas perigosas ou ameaçadoras (AMERICAN PSYCHIATRIC ASSOCIATION, 2015; CASTILHO et al., 2000). Ela pode ser decorrente tanto de uma predisposição neurobiológica herdada (traço de ansiedade) quanto de uma desordem emocional temporária (estado de ansiedade) (ROSEN; SCHILKIN, 1998). Assim como a ira, todas as pessoas experimentam diferentes níveis de ansiedade ao longo da vida. Entretanto, ela passa ao nível de patologia quando os seus efeitos se tornam exagerados, desproporcionais em relação ao estímulo, influenciando negativamente nas funções cognitivas, no conforto emocional e no bemestar do indivíduo (CASTILHO et al., 2000). Como consequências, a ansiedade por afetar as características cognitivas, condutuais e somáticas do indivíduo, provocando nele tensão, falta de comportamentos adaptativos e ativação do sistema nervoso. As principais reações fisiológicas causadas pela ansiedade são sudorese, tremores, cansaço, alterações no sono, falha de memória, baixa autoestima e aumento da frequência cardíaca (AMERICAN PSYCHIATRIC ASSOCIATION, 2015; CERQUEIRA; ZORZAL; ÁVILA, 2012; KENNY, 2011).

Finalmente, é importante frisar que quando uma pessoa possui um estado de ânimo negativo, os pensamentos que surgem na sua mente tendem a ser congruentes com o seu estado de ânimo. De acordo com Chen, Zhou e Bryant (2007), os estados de ânimo negativos provocam cognições desagradáveis que induzem sentimentos de angústia. Para 
aliviar, regular ou melhorar tais estados de ânimo, os indivíduos tendem a utilizar diferentes tipos de recursos, sendo a música um daqueles de maior recorrência.

\section{A pandemia da COVID-19}

Considerando as recentes transformações ocasionadas pela disseminação da COVID-19 no mundo, muitas pessoas se veem cada vez mais imersas em um ambiente de grande preocupação, tristeza e insegurança. No mundo, atualmente já são 70 milhões de pessoas infectadas, resultando em aproximadamente 1,6 milhões de vidas perdidas em razão deste vírus ${ }^{1}$. Essa pandemia tem promovido uma forte mudança nos panoramas sanitário, econômico e, consequentemente, social em todos os países, com maior impacto sobre aqueles indivíduos que se encontram em situação de vulnerabilidade social (BAQUI et al., 2020).

O Brasil registrou o seu primeiro caso de COVID-19 no dia 26 de fevereiro de 2020 e, desde então, vem sofrendo bastante com o avanço acelerado da pandemia. Grandes capitais (ex.: Manaus e São Luís) registraram o colapso dos seus sistemas de saúde, com a superlotação das Unidades de Terapia Intensiva (UTI) e a ausência de respiradores para realizar o atendimento daqueles indivíduos que apresentavam sintomas mais graves da doença (RIBEIRO; LIMA; WALDMAN, 2020). Para Ortega e Orsini (2020), as ações do governo federal desde o início da pandemia se mostraram ineficientes e incongruentes com as orientações dos profissionais da saúde e da Organização Mundial da Saúde (OMS). Segundo os autores, a adoção de uma política de negação da ciência, de um governo de exceção e de uma ignorância estratégica tem levado o Brasil a um estado de calamidade, atingindo em dezembro a marca de 7 milhões de infectados e 180 mil mortos, sendo a maioria destes pessoas negras e moradoras da região norte do país (BAQUI et al., 2020).

A ausência de um plano nacional de combate ao Coronavírus fez com que Estados e Municípios, cada um à sua maneira, implementassem estratégias diversificadas. Até o momento da redação deste artigo, poucas foram as cidades que realizaram lockdown, uma medida extremada de isolamento social. A grande maioria optou por medidas mais brandas para a contenção do vírus, como distanciamento social, rastreamento de

\footnotetext{
${ }^{1}$ Para maiores informações, acesse: https://www.worldometers.info/coronavirus/.
} 
deslocamento da população, utilização de máscaras e álcool em gel, além de campanhas de conscientização sobre higiene pessoal (LIMA-COSTA et al., 2020). Durante os períodos mais críticos da pandemia, foram criadas medidas restritivas para evitar aglomerações, tais como proibição de eventos sociais e esportivos, delimitação de normas rigorosas para funcionamento de bares e restaurantes, e fechamento de casas de shows, teatros e cinemas (GARCIA; DUARTE, 2020). Escolas e universidades adotaram o ensino remoto em substituição às aulas presenciais, uma medida bastante criticada devido à desigualdade de condições de acesso ao conteúdo pelos discentes, bem como à falta de capacitação dos docentes para se adequarem a essa nova demanda (MÉDICI; TATTO; LEÃO, 2020). Além disso, diversas empresas implementaram o formato denominado home-office, isto é, a transferência das atividades laborais para o ambiente familiar, acabando com a concepção de privacidade e flexibilizando (ou quase inexistindo) as delimitações sobre os horários de trabalho (FILGUEIRAS; STULTS-KOLEHMAINEN, 2020; GARCIA; DUARTE, 2020; MAIA; DIAS, 2020).

Para amenizar os impactos do Coronavírus, o Governo Federal adotou algumas medidas importantes (ainda que limitadas). No âmbito econômico, foi implementado a partir do mês de maio de 2020 um programa emergencial de auxílio financeiro destinado à população mais vulnerável, atingindo um público aproximado de 50 milhões de brasileiros (CARDOSO, 2020). No âmbito educativo, foram oferecidos pacotes de dados móveis para estudantes de instituições públicas em situação de vulnerabilidade, para que pudessem acessar as aulas (ARRUDA, 2020). Na saúde, Estados e Municípios compraram respiradores, máscaras e outros insumos (com indícios de superfaturamento), criando também hospitais de campanha para atendimento da população durante os períodos mais críticos da doença (SILVA; LIMA, 2020). Atualmente, o Brasil está entrando na segunda onda da pandemia e pesquisadores receiam que isso pode piorar ainda mais a situação do país (FERRANTE et al., 2020).

Diferentes estudos têm constatado que momentos de crise, como o atual, tendem a gerar um forte impacto psicológico na população, aumentando significativamente a recorrência e a intensidade das emoções negativas (KEOGH et al., 2004; KOUZMA; KENNEDY, 2004; PUTWAIN, 2009). Em pesquisa realizada entre os meses de abril e maio de 2020, o Ministério da Saúde do Brasil verificou que 86,5\% dos participantes apresentaram transtorno de ansiedade, 45,5\% apresentaram transtorno de estresse pós- 
traumático e 16,0\% indicaram sintomas de depressão ${ }^{2}$. Em estudo recente, Maia e Dias (2020) avaliaram estudantes universitários antes e após a pandemia iniciar, encontrando um aumento considerável dos níveis de estresse, ansiedade e depressão durante o período pandêmico. Assim sendo, torna-se urgente e necessário a adoção de medidas para a redução de tais impactos, oportunizando uma melhora significativa no humor, na qualidade de vida e, consequentemente, no bem-estar das pessoas.

Nesse sentido, a música pode ser considerada uma ferramenta fundamental para auxiliar a superar essa situação. Segundo McFerran et al. (2015), indivíduos que apresentam níveis elevados de estados emocionais negativos necessitam de atividades com maior potencial de desenvolvimento motivacional para atiçarem estados emocionais positivos. Dessa maneira, pesquisas têm comprovado que atividades musicais tanto de escuta quanto de execução, realizadas de forma individual ou coletiva, podem resultar em uma melhora significativa no estado de ânimo das pessoas (LUDKE; FERREIRA; OVERY, 2013). Assim sendo, será exposta a continuação uma revisão da literatura sobre o uso da música como ferramenta eficaz para a regulação do estado de ânimo.

\section{Música e estado de ânimo}

A música é uma arte que chega ao íntimo das pessoas, provocando nelas uma variedade de emoções e fomentando sua liberação afetiva, emocional e intelectual (LÁCARCEL, 2003). Ao longo da história, diferentes valores têm sido outorgados à música e cada sociedade a tem utilizado em função das características do seu contexto sociocultural. Desde a Idade Média, diversos estudos concederam a essa arte um valor terapêutico, dada sua grande utilidade no tratamento de alterações psicológicas, tais como a melancolia e a depressão. Posteriormente, a partir do século XIX, o uso da música para essa finalidade cresceu e se converteu muito popular em função das melhorias produzidas nos estados mentais de pacientes (GARRIDO; DAVIDSON, 2013).

Baseado nesse panorama histórico, é possível destacar o crescimento quantitativo de pesquisas interessadas em conhecer os possíveis efeitos da música na indução do estado de ânimo. Assim, algumas correntes de estudos relacionados à música (como a

\footnotetext{
${ }^{2}$ Para maiores informações, acesse: https://antigo.saude.gov.br/noticias/agencia-saude/47527-ministerioda-saude-divulga-resultados-preliminares-de-pesquisa-sobre-saude-mental-na-pandemia.
} 
Psicologia da Música e a Musicoterapia) tratam especificamente sobre a conexão entre mente e corpo, analisando por variados pontos de vista as reações - psicológicas e fisiológicas - do corpo humano frente à estimulação musical (GARRIDO; DAVIDSON, 2013). Um exemplo disso é o estudo de Vastfjall (2002), que identificou variações no estado de ânimo dos participantes em função do tipo de música que escutavam (alegres, tristes ou desconhecidas). Por sua vez, existem evidências que sugerem a eficiência do uso da música para reduzir estresse e no tratamento da depressão (AALBERS et al., 2008).

Diferentes trabalhos apontam que uma das funções que mais motivam as pessoas a escutar música é sua atuação como estabilizadora e reguladora do estado de ânimo (MCFERRAN et al., 2015; RENTFROW; GOSLING, 2003; SCHÄFER; SEDLMEIER, 2009). Seu uso na vida diária não parece ter relação com tocar um instrumento de maneira profissional ou com considerar a música simplesmente como um hobby, mas sim relacionada com questões afetivas e com um maior compromisso musical-pessoal através da sua escuta (SAARIKALLIO; NIEMINEN; BRATTICO, 2013). Estudos apontam que a escuta musical pode servir como meio de relaxamento e fuga dos problemas diários, para criar uma atmosfera de diversão em uma festa, ou para obter fortes sensações emocionais (HERRERA; SOARES-QUADROS JR.; LORENZO, 2018; SCHÄFER; SEDLMEIER, 2009). Por isso, é possível supor que a real importância da música na regulação do estado de ânimo seja resultado da sua versatilidade para satisfazer múltiplas necessidades e objetivos (SAARIKALLIO; ERKKILÄ, 2007).

Sob um ponto de vista neurológico, Blood e Zatorre (2001) acreditam que a influência da música no estado de ânimo pode estar relacionada com as regiões cerebrais que são afetadas durante a realização de uma atividade musical. Benedito (2010) afirma que as características da música (ritmo, entonação, melodia, altura, instrumentação, duração e harmonia) repercutem diretamente em funções ligadas ao sistema nervoso central, através da neurotransmissão cerebral, provocando determinados estados anímicos nos indivíduos. Além disso, esses elementos musicais ocupam um importante papel nas funções fisiológicas e psicológicas das pessoas criando alterações nos estados de ânimo. Outros autores acreditam que as experiências musicais de ouvir ou fazer música recrutam tanto regiões corticais do cérebro (ex.: córtex pré-frontal, córtex pré-motor, córtex motor, etc.), quanto as estruturas do sistema límbico e paralímbico 
(incluindo a amígdala e o tálamo), regiões similares àquelas associadas ao prazer e à recompensa (HUSAIN et al., 2002; KOELSCH, 2010). Husain et al. (2002), por sua vez, afirmam que a variação rítmica de uma canção pode provocar atividade no córtex motor esquerdo, área responsável pela excitação e que exerce um importante papel na regulação do estado de ânimo.

Baseado nas evidências anteriormente apresentadas e relacionando com os impactos psicológicos provocados pela pandemia da COVID-19, torna-se fundamental buscar na literatura científica informações sobre gêneros musicais utilizados para a indução de estados de ânimo positivos. Hakanen (1995) observou em seu estudo a existência de uma correlação positiva entre a preferência por rap e emoções positivas como felicidade e excitação. Kim e Kim (2007) encontraram em seu estudo que a escuta de hip-hop produziu uma diminuição da angústia psicológica e da fadiga entre jovens, aparecendo associada positivamente ao bem-estar. Outros autores têm encontrado evidências entre a escuta de música clássica e o aumento nos níveis de relaxamento, concentração, calma, estimulação da criatividade e da imaginação (ORDOÑEZ et al., 2011; REA; MACDONALD; CARNES, 2010). O estudo de McFerran et al. (2015) apontou que dance foi o gênero musical que correlacionou mais fortemente com estados emocionais positivos.

Para além dos estudos correlacionais, muitos pesquisadores têm se interessado pelos efeitos fisiológicos provocados pela música, o que poderia ajudar a potencializar o bem-estar. Existem evidências do uso da música para a indução de respostas orgânicas de forma involuntária, como mudanças na pressão sanguínea, nas frequências respiratórias e no ritmo cardíaco (BENEDITO, 2010; CUSTODIO; CANO-CAMPOS, 2017). Outros autores afirmam que a música pode ter um forte efeito na resposta galvânica da pele, processo fisiológico utilizado para medir a atividade elétrica produzida por alterações psicológicas tais como emoções e pensamentos, auxiliando na identificação de situações que causam estresse e ansiedade (BERRIO; HERRERA, 2014). 


\section{Considerações finais}

Tendo em mente o contexto atual, em direção à pós-pandemia, é importante buscar formas de reduzir os níveis dos variados tipos de estados emocionais negativos que constituem o nosso perfil psicológico e, como visto anteriormente, a música aparece como um instrumento de alto potencial para a regulação emocional. Com isso, é possível conceber a música como uma medida de grande eficácia e de baixo custo para a incitação de estados de ânimo positivos, proporcionando consequentemente uma melhoria na sensação de bem-estar e na qualidade de vida das pessoas. Assim, espera-se que esse estudo possa incentivar e contribuir para o desenvolvimento de novos estudos acerca da relação entre música e humor.

\section{Referências}

AALBERS, Sonja; FUSAR-POLI, Laura; FREEMAN, Ruth; SPREEN, Marinus; KET, Johannes; VINK, Annemiek; MARATOS, Ana; CRAWFORD, Mike; CHEN, Xi-Jing; GOLD, Christian. Music therapy for depression. Cochrane Database of Systematic Reviews, 1, CD004517, nov. 2017. Disponível em: http://dx.doi. org/ 10.1002/14651858.CD004517.pub3.

ALI, S. Omar; PEYNIRCIOĞLU, Zehra. Songs and emotions: are lyrics and melodies equal partners? Psychology of Music, v. 34, n. 4, p. 511-534, out. 2006. Disponível em: https://www.doi.org/ 10.1177/0305735606067168.

ALMEIDA, Ana. A emoção na sala de aula. 8. ed. São Paulo: Papirus, 1999.

AMERICAN PSYCHIATRIC ASSOCIATION. Manual diagnóstico e estatístico de transtornos mentais: DSM-5. 5. ed. Porto Alegre: Artmed, 2015.

ARGYLE, Michael; MARTIN, Maryanne. The psychological cause of Happiness. In: STRACK, Fritz; ARGYLE, Michael; SCHWARZ, Nobert. Subjective well-being: an interdisciplinary perspective. Oxford: Pergamon Press, 1991. p. 77-101.

ARRUDA, Eucidio. Educação remota emergencial: elementos para as políticas públicas na educação brasileira em tempos de COVID-19. EmRede: Revista de Educação à distância, v. 7, n. 
1, p. 257-275, mai. 2020. Disponível em: https://www.aunirede.org.br/revista/index.php/emrede/article/view/621.

BAQUI, Pedro; MPHIL, Ioana; MARRA, Valerio; ERCOLE, Ari; SCHAAR, Mihaela. Ethnic and regional variations in hospital mortality from COVID-19 in Brazil: a cross-sectional observational study. The Lancet Global Health, v. 8, n. 8, p. e1018-e1026, jul. 2020. Disponível em: https://doi.org/10.1016/S2214-109X(20)30285-0.

BENEDITO, María. Reflexiones en torno a la utilidad de la música en la terapia psicológica con adolescentes. Revista Española de Pediatría, v. 66, n. 2, p. 136-140, 2010.

BERRIO, Nelson; HERRERA, Lucía. Respuestas psicofisiológicas ante la escucha de diferentes géneros musicales de contenido religioso-cristiano. DEDiCA, v. 5, p. 179-196, mar. 2014. Disponível em: https://revistaseug.ugr.es/index.php/dedica/article/view/7008.

BLOOD, Anne; ZATORRE, Robert. Intensely pleasurable responses to music correlate with activity in brain regions implicated in reward and emotion. Proceedings of the National Academy of Sciences of the United States of America, v. 98, p. 11818-11823, set. 2001. Disponível em: https://doi.org/10.1073/pnas.191355898.

BOTH, Fiemke; HOOGENDOORN, Mark; KLEIN, Michael; TREUR, Jan. (2008). Modeling the dynamics of mood and depression. In: GHALLAB, M.; SPYROPOULOS, C. D.; FAKOTAKIS, N.; AVOURIS, N. Proceedings of the 18th European Conference on Artificial Intelligence, ECAI'08. Grécia: IOS Press, 2008. p. 266-270. Disponível em: https://www.doi.org/10.3233/978-1-58603-891-5-266.

CAMPOS, Josiane; DEL-PRETTE, Almir; DEL-PRETTE, Zilda. Depressão na adolescência: habilidades sociais e variáveis sociodemográficas como fatores de risco/proteção. Estudos e Pesquisas em Psicologia, Rio de Janeiro, v. 14, n. 2, p. 408-428, 2014. Disponível em: https://doi.org/10.12957/epp.2014.12645.

CARDOSO, Bruno. The implementation of emergency aid as an exceptional measure of social protection. Brazilian Journal of Public Administration, v. 54, n. 4, p.1052-1063, ago. 2020. Disponível em: https://www.doi.org/10.1038/s41591-020-1026-x.

CASTILHO, Ana; RECONDO, Rogéria; ASBAHR, Fernando; MANFRO, Gisele. Transtornos de ansiedade. Revista Brasileira de Psiquiatria, São Paulo, v. 22, supl. 2, p. 20-23, dez. 2000. Disponível em: http://dx.doi.org/10.1590/S1516-44462000000600006.

CERQUEIRA, Daniel; ZORZAL, Ricieri; ÁVILA, Guilherme. Considerações sobre a aprendizagem da performance musical. Per Musi, Belo Horizonte, n. 26, p. 94-109, dez. 2012. Disponível em: http://dx.doi.org/10.1590/S1517-75992012000200010.

CHEN, Lei; ZHOU, Shuhua; BRYANT, Jennings. Temporal changes in mood repair through music consumption: Effects of mood, mood salience, and individual differences. Media Psychology, v. 9, n. 3, p. 695-713, dez. 2007. Disponível em: https://doi.org/10.1080/15213260701283293.

CUSTODIO, Nilton; CANO-CAMPOS, María. Efectos de la música sobre las funciones cognitivas. Revista de Neuro-Psiquiatría, v. 80, n. 1, p. 60-69, abr. 2017. Disponível em: https://doi.org/10.20453/rnp.v80i1.3060. 
DALGALARRONDO, Paulo. Psicopatologia e semiologia dos transtornos mentais. Artmed. Porto Alegre: 2018.

DEL BARRIO, Victoria; ALUJA, Antón; SPIELBERGER, Charles. STAXI-NA: Inventario de expresión de ira estado - rasgo en niños y adolescentes. 2. ed. Madrid: Tea Ediciones, 2009.

DJAMASBI, Soussan; STRONG, Diane; DISHAW, Mark. Affect and acceptance: Examining the effects of positive mood on the technology acceptance model. Decision Support Systems, v. 48, n. 2, p. 383-394, jan. 2010. Disponível em: https://doi.org/10.1016/j.dss.2009.10.002.

ECHEVERRÍA, Rafael. Emociones y estados de ánimo. In: ECHEVERRÍA, R. Ontología del lenguaje. Chile: Lom Ediciones S.A., 2005. p. 152-173.

ETZEL, Joset; JOHNSEN, Erica; DICKERSON, Julie; TRANEL, Daniel; ADOLPHS, Ralph. Cardiovascular and respiratory responses during musical mood induction. International Journal of Psychophysiology, v. 61, n. 1, p. 57-69, fev. 2006. Disponível em: https://doi.org/10.1016/j.ijpsycho.2005.10.025.

FERRANTE, Lucas; STEINMETZ, Wilhelm; ALMEIDA, Alexandre; LEÃO, Jeremias; VASSÃO, Ruth; TUPINAMBÁS, Unaí; FEARNSIDE, Philip; DUCZMAL, Luiz. (2020). Brazil's policies condemn Amazonia to the second wave of COVID-19. Nature Medicine, v. 26, p. 1315, set. 2020. https://doi.org/10.1038/s41591-020-1026-x.

FILGUEIRAS, Alberto; STULTS-KOLEHMAINEN, Matthew. The relationship between behavioural and psychosocial factors among Brazilians in quarantine due to COVID-19. The Lancet, 2020. (no prelo). Disponível em: http://dx.doi.org/10.2139/ssrn.3566245.

FIORIN, José. Paixões, afetos, emoções e sentimentos. CASA: Cadernos de Semiótica Aplicada, v. 5, n. 2, p. 541-555, mar. 2008. Disponível em: https://doi.org/10.21709/casa.v5i2.

FRIJDA, Nico. The emotions. Nova Iorque (EUA): Cambridge University Press, 1986.

GALLARDO VERGARA, René. Naturaleza del estado de ánimo. Revista Chilena de Neuropsicología, v. 1, n. 1, p. 29-40, dez. 2006. Disponível em: http://www.redalyc.org/articulo.oa?id=179317886004.

GARCIA, Leila; DUARTE, Elisete. Nonpharmaceutical interventions for tackling the COVID19 epidemic in Brazil. Epidemiologia e Serviços de Saúde, v. 29, n. 2, p. e2020222, abr. 2020. Disponível em: https://doi.org/10.5123/s1679-49742020000200009.

GARRIDO, Sandra; DAVIDSON, Jane. Music and mood regulation: A historical enquiry into individual differences and musical prescriptions through the ages. Australian Journal of Music Therapy, v. 24, p. 89-109, $2013 . \quad$ Disponível em: https://search.proquest.com/docview/1463029177?accountid=14542.

GARRIDO, Sandra. A systematic review of the studies measuring mood and emotion in response to music. Psychomusicology: Music, Mind, and Brain, v. 24, n. 4, p. 316-327, 2014. Disponível em: https://doi.org/10.1037/pmu0000072.

HAKANEN, Ernest. Emotional use of music by African American adolescents. Howard Journal of Communications, v. 5, n. 3, p. 214-222, fev. 1995. Disponível em: https://doi.org/10.1080/10646179509361664. 
HERRERA, Lucía; SOARES-QUADROS JR., João; LORENZO, Oswaldo. Music preferences and personality in Brazilians. Frontiers in Psychology, v. 9, p. 1488, ago. 2018. Disponível em: https://doi.org/10.3389/fpsyg.2018.01488.

HUSAIN, Gabriela; THOMPSON, William; SCHELLENBERG, E. Gleen. Effects of musical tempo and mode on arousal, mood, and spatial abilities. Music Perception: An Interdisciplinary Journal, v. 20, n. 2, p. 151-171, 2002. http://dx.doi.org/10.1525/ mp.2002.20.2.151.

ISEN, Alice. Some ways in which positive affect influences decision making and problem solving. In: LEWIS, M.; HAVILAND-JONES, J.; FELDMAN, L. Handbook of Emotions. Nova Iorque (EUA): The Guilford Press, 2008. p. 548-573.

JUSLIN, Patrick; SLOBODA, John. Handbook of music and emotion: theory, research and applications. Nova Iorque (EUA): Oxford University Press, 2010.

JUSLIN, Patrick; VÄSTFJÄLL, Daniel. Emotional responses to music: The need to consider underlying mechanisms. Behavioral and Brain Sciences, v. 31, n. 5, p. 559-575, out. 2008. Disponível em: https://doi.org/10.1017/S0140525X08005293.

KENNY, Diane. The psychology of music performance anxiety. Nova Iorque (EUA): Oxford University Press, 2011.

KEOGH, Edmund; BOND, Frank; FRENCH, Christoph; RICHARDS, Anne; DAVIS, Robert. Test anxiety, susceptibility to distraction and examination performance. Anxiety, Stress and Coping, v. 17, n. 3, p. 241-252, jan. 2004. Disponível em: https://doi.org/10.1080/10615300410001703472.

KIM, Sungwoon; KIM, Jingu. Mood after various brief exercise and sport modes: aerobics, hiphop dancing, ice skating, and body conditioning. Perceptual and Motor Skills, v. 104, p. 12651270, jun. 2007. Disponível em: https://doi.org/10.2466/pms.104.4.1265-1270.

KOELSCH, Stefan. Towards a neural basis of music-evoked emotions. Trends in Cognitive Sciences, v. 14, p. 131-137, fev. 2010. Disponível em: https://doi.org/10.1016/j.tics.2010.01.002.

KOUROS, Chrystyna; EL-SHEIKH, Mona. Daily mood and sleep: reciprocal relations and links with adjustment problems. Journal of Sleep Research, v. 24, n. 1, p. 24-31, set. 2015. Disponível em: https://doi.org/10.1111/jsr.12226.

KOUZMA, Nadya; KENNEDY, Gerard. Self-reported sources of stress in senior high school students. Psychological Reports, v. 94, n. 1, p. 314-316, fev. 2004. Disponível em: https://doi.org/10.2466/pr0.94.1.314-316.

LAGOS SAN MARTÍN, Nelly; GARCÍA-FERNÁNDEZ, José; INGLÉS SAURA, Cándido; GONZÁLVEZ-MACIÁ, Carolina; VICENT-JUAN, Maria. Capacidad predictiva de la ira sobre la ansiedad escolar en estudiantes chilenos de Educación Secundaria. International Journal of Developmental and Educational Psychology, v. 1, n. 1, p. 389-395, 2015. Disponível em: https://www.redalyc.org/articulo.oa?id=349851779039.

LEITE, Sérgio; TASSONI, Elvira. A afetividade em sala de aula: as condições de ensino e a mediação do professor. In: AZZI, R.; SADALLA, A. Psicologia e formação docente: desafios e conversa. São Paulo: Casa do Psicólogo, 2002. p. 113-142. 
LIMA-COSTA, Maria; MAMBRINI, Juliana; ANDRADE, Fabíola; PEIXOTO, Sérgio; MACINKO, James. Social distancing, use of face masks and hand washing among participants in the Brazilian longitudinal study of aging: the ELSI-COVID-19 initiative. Cadernos de Saúde Pública, v. 36, supl. 3, p. e00193920, out. 2020. Disponível em: https://doi.org/10.1590/0102$311 \mathrm{X} 00193920$.

LONGUI, Magalí; BEHAR, Patrícia; BERCHT, Magda. Os fatores motivacionais e os estados de ânimo em ambientes virtuais de aprendizagem. In: CONGRESSO IBEROAMERICANO DE INFORMÁTICA EDUCATIVA, 1., 2010, Santiago (Chile). Anais... Santiago (Chile): Universidad de Chile, 2010. p. 551-558.

LUDKE, Karen; FERREIRA, Fernanda; OVERY, Katie. Singing can facilitate foreign language learning. Memory \& Cognition, v. 42, p. 41-52, jul. 2014. Disponível em: https://doi.org/10.3758/s13421-013-0342-5.

MAIA, Berta; DIAS, Paulo. Ansiedade, depressão e estresse em estudantes universitários: o impacto da COVID-19. Estudos de Psicologia, Campinas, v. 37, p. e200067, mai. 2020.

MCFERRAN, Katrina; GARRIDO, Sandra; O'GRADY, Lucy; GROCKE, Denise; SAWYER, Susan. Examining the relationship between self-reported mood management and music preferences of Australian teenagers. Nordic Journal of Music Therapy, v. 24, n. 3, p. 187-203, 2015. Disponível em: https://doi.org/10.1080/08098131.2014.908942.

MÉDICI, Mônica; TATTO, Everson; LEÃO, Marcelo. Percepções de estudantes do Ensino Médio das redes pública e privada sobre atividades remotas ofertadas em tempos de pandemia do coronavírus. Revista Thema, v. 18, n. especial, p. 136-155, ago. 2020. Disponível em: https://doi.org/10.15536/thema.V18.Especial.2020.136-155.1837.

NOGUEIRA, Bruno; LEITE, Sérgio. A afetividade no processo de orientação de pesquisa científica. Revista de Educação da PUC-Campinas, v. 19, n. 3, p. 249-259, abr. 2015. Disponível em: https://doi.org/10.24220/2318-0870v19n3a2852.

OATLEY, Keith; JOHNSON-LARID, Philip. Towards a cognitive theory of emotions. Cognition and Emotion, v. 1, n. 1, 1987. Disponível em: https://doi.org/10.1080/02699938708408362.

OLIVA MENDOZA, Félix; HERNÁNDEZ POZO, María; CALLEJA BELLO, Nazira. Validación de la versión mexicana del inventario de expresión de ira estado-rasgo (STAXI-2). Acta Colombiana de Psicología, v. 13, v. 2, p. 107-117, dez. 2010. Disponível em: http://www.scielo.org.co/scielo.php?script=sci_arttext\&pid=S0123-

$91552010000200010 \& \operatorname{lng}=$ en\&nrm $=$ iso.

ORDOÑEZ, Esteban; SÁNCHEZ, Jaime; SÁNCHEZ, Marco; ROMERO, Christian; BERNAL, Juan. Análisis del Efecto Mozart en el desarrollo intelectual de las personas adultas y niños. Ingenius, v. 5, p. 45-54, jan./jun. 2011. Disponível em: https://dialnet.unirioja.es/servlet/articulo? codigo $=5972769$.

ORGANIZAÇÃO MUNDIAL DA SAÚDE. Depression and other common mental disorders: Global health estimates. Geneva (Suíça): WHO, 2017.

ORTEGA, Francisco; ORSINI, Michael. Governing COVID-19 without government in Brazil: Ignorance, neoliberal authoritarianism, and the collapse of public health leadership. Global Public Health, v. 15, n. 9, p. 1257-1277, jul. 2020. Disponível em: https://doi.org/10.1080/17441692.2020.1795223. 
PERGHER, Giovanni; GRASSI-OLIVEIRA, Rodrigo; AVILA, Luciana; STEIN, Lilian. Memória, humor e emoção. Revista de Psiquiatria do Rio Grande do Sul, Porto Alegre, v. 28, n. 1, p. 61-68, jan./abr. 2006. Disponível em: http://dx.doi.org/10.1590/S010181082006000100008 .

PUTWAIN, David. Situated and contextual features of test anxiety in UK adolescent students. School Psychology International, v. 30, n. 1, p. 56-74, fev. 2009. Disponível em: https://doi.org/10.1177/0143034308101850.

REA, Christopher; MACDONALD, Pamelyn; CARNES, Gwen. Listening to classical, pop, and metal music: an investigation of mood. Emporia State Research Studies, v. 46, n. 1, p. 1-3, jan. 2010. Disponível em: https://esirc.emporia.edu/handle/123456789/381.

RENTFROW, Peter; GOSLING, Samuel. The do re mi's of everyday life: The structure and personality correlates of music preferences. Journal of Personality and Social Psychology, v. 84, n. 6, p. 1236-1256, jun. 2003. Disponível em: https://www.doi.org/ 10.1037/00223514.84.6.1236.

RIBEIRO, Helena; LIMA, Viviana; WALDMAN, Eliseu. In the COVID-19 pandemic in Brazil, do brown lives matter? The Lancet Global Health, v. 8, n. 8, p. e-976-e977, jul. 2020. Disponível em: https://doi.org/10.1016/S2214-109X(20)30314-4.

ROSEN, Jeffrey; SCHILKIN, Jay. From normal fear to pathological anxiety. Psychological Review, v. 105, p. 325-50, abr. 1998. Disponível em: https://doi.org/10.1037/0033$295 \times .105 .2 .325$.

ROY, Mathieu; PERETZ, Isabelle; RAINVILLE, Pierre. Emotional valence contributes to musicinduced analgesia. Pain, v. 134, n. 1-2, p. 140-147, jan. 2008. Disponível em: https://doi.org/10.1016/j.pain.2007.04.003.

RUFINO, Sueli; LEITE, Ricardo; FRESCHI, Larissa; VENTURELLI, Vanessa; OLIVEIRA, Elizabeth; MASTROROCCO FILHO, Diogo. Aspectos gerais, sintomas e diagnóstico da depressão. Revista Saúde em Foco, n. 10, p. 837-843, 2018.

SAARIKALLIO, Suvi; ERKKILÄ, Jaakko. The role of music in adolescents' mood regulation. Psychology of Music, v. 35, n. 1, p. 88-109, jan. 2007. Disponível em: https://doi.org/10.1177/0305735607068889.

SAARIKALLIO, Suvi; NIEMINEN, Sirke; BRATTICO, Elvira. Affective reactions to musical stimuli reflect emotional use of music in everyday life. Musicae Scientiae, v. 17, n. 1, p. 27-39, out. 2013. Disponível em: https://doi.org/10.1177/1029864912462381.

Saarikallio, Suvi. Development and validation of the brief music in mood regulation scale (BMMR). Music Perception: an Interdisciplinary Journal, v. 30, n. 1, p. 97-105, 2012. Disponível em: https://doi.org/10.1525/mp.2012.30.1.97.

SANZ, Jesús. Un instrumento para evaluar la eficacia de los procedimientos de inducción de estado de ánimo: "La Escala de Valoración del Estado de Ánimo" (EVEA). Análisis y Modificación de Conducta, v. 27, n. 111, p. 71-110, 2001. Disponível em: https://dialnet.unirioja.es/servlet/articulo?codigo $=7061179$. 
SCHÄFER, Thomas; SEDLMEIER, Peter. From the functions of music-to-music preference. Psychology of Music, v. 37, n. 3, p. 279-300, mar. 2009. Disponível em: https://doi.org/10.1177/0305735608097247.

SEIDEL, Angelika; PRINZ, Jesse. Mad and glad: Musically induced emotions have divergent impact on morals. Motivation and Emotion, v. 37, n. 3, p. 629-637, set. 2013. Disponível em: https://doi.org/10.1007/s11031-012-9320-7.

SILVA, Josimário; LIMA, Andressa. Principais consequências bioéticas e jurídicas do COVID19 no Brasil. Revista Española de Bioética, n. 54, p. 36-48, dez. 2020. Disponível em: https://revistaeidon.es/index.php/revistaeidon/article/view/131.

TAMIR, Maya; ROBINSON, Michael. The happy spotlight: Positive mood and selective attention to rewarding information. Personality and Social Psychology Bulletin, v. 33, n. 8, p. 1124-1136, ago. 2007. Disponível em: https://doi.org/10.1177/0146167207301030.

WALSH, Lisa; BOEHM, Julia; LYUBOMIRSKY, Sonja. Does happiness promote career success? Journal of Career Assessment, v. 16, n. 1, p. 101-116, 2008. Disponível: https://doi.org/10.1177/1069072717751441.

WRÓBEL, Monika; KRÓLEWIAK, Klara. Is your mood more contagious if you are likeable? The role of liking in the social induction of affect. Polish Psychological Bulletin, v. 46, n. 3, p. 413-420, abr. 2015. Disponível em: https://doi.org/10.1515/ppb-2015-0048.

ZATORRE, Robert; SALIMPOOR, Valorie. From perception to pleasure: music and its neural substrates. Proceedings of the National Academy of Sciences, v. 110, supl. 2, p. 10430-10437, jun. 2013. Disponível em: https://doi.org/10.1073/pnas.1301228110. 
